\title{
ALGEBRAIC NUMBERS AND TOPOLOGICALLY EQUIVALENT MEASURES IN THE CANTOR SET
}

\author{
K. J. HUANG
}

\begin{abstract}
It is known that the transcendental and rational numbers in the unit interval are not binomial numbers. In this article we will show that the algebraic integers of degree 2 are not binomial numbers either. Therefore two shift invariant measures $u(s), u(r)$ with $r$ being an algebraic integer of degree 2 in the unit interval are topologically equivalent if and only if $s=r$ or $s=1-r$. We also show that for each positive integer $n>2$, there are algebraic integers and fractionals of degree $n$ in the unit interval that are binomial numbers.
\end{abstract}

Let $X$ be a topological space. Two Borel measures $u$ and $v$ are said to be topologically equivalent whenever $u=v h$ for some homeomorphism $h$ of $X$ onto itself. This notion sets up an equivalence relation which partitions the family of Borel measures into disjoint classes. By restricting attention, if necessary, to a suitably defined subfamily of measures, one can ask for the number of equivalence classes. One can also try to uncover necessary conditions which intrinsically characterize measures which belong to the same class.

Topologically equivalent measures in the $n$-dimensional unit cube, the space of irrational numbers in the unit interval, and the Hilbert cube have been studied, respectively, by Oxtoby and Ulam [1], Oxtoby [2], and by Oxtoby and Prasad [3]. In [4] F. J. Navarro-Bermúdez studied the topologically equivalent measures in the Cantor space.

Let $X$ be the Cantor space of infinite product of $X_{n}$ where $n=1,2, \ldots$ and $X_{n}=\{0,1\}$ with discrete topology. Set

$$
\left[i_{1}, i_{2}, \ldots, i_{m}\right]=\left\{\left(x_{j}\right): x_{j}=i_{j} \text { for } j=1, \ldots, m \text {, and }\left(x_{j}\right) \text { in } X\right\} .
$$

These sets form a base for the topology of $X$. Two of these sets $\left[i_{1}, \ldots, i_{m}\right]$ and $\left[j_{1}, \ldots, j_{m}\right]$ are equal or disjoint, depending on whether the $m$-tuples $\left(i_{1}, \ldots, i_{m}\right)$ and $\left(j_{1}, \ldots, j_{m}\right)$ are identical or not.

We restrict our attention to the family $F$ of probability measures in $X$ which consists of product measures $u(r)=\prod_{n=1}^{\infty} u(n)$ subject to the condition

$$
r=u(n)(1)=u(n+1)(1)>0 \text { for all } n,
$$

where $r$ is in the unit interval $[0,1]$. Among the probability measures on $X$, these are precisely those which are invariant under the Bernoulli shift transformation $T$ on $X$. A Borel measure $u$ on $X$ is invariant under $T$ if $u\left(T^{-1}(U)\right)=u(U)$ for every Borel set $U$ of $X$.

Two numbers $r$ and $s$ in the unit interval $[0,1]$ are said to be binomially related whenever (1), (2) hold: for some positive integers $m, n$ and nonnegative integers

Received by the editors October 31, 1984 and, in revised form, April 25, 1985.

1980 Mathematics Subject Classification. Primary 12A15. 
$a_{i}, b_{j}$ with $0 \leq a_{i} \leq\left(\begin{array}{c}m \\ i\end{array}\right)$ and $0 \leq b_{j} \leq\left(\begin{array}{c}n \\ j\end{array}\right)$, where $i=0, \ldots, m$ and $j=0,1, \ldots, n$, and we have

$$
\begin{aligned}
& s=\sum_{0}^{m} a_{i} r^{m-i}(1-r)^{i}, \\
& r=\sum_{0}^{n} b_{j} s^{n-j}(1-s)^{j} .
\end{aligned}
$$

In [4] F. J. Navarro-Bermúdez proved that if $u(r)$ is topologically equivalent to $u(s)$, then $r$ is binomially related to $s$. He also proved that for each transcendental or rational $r$ in the unit interval, if $u(s)$ is topologically equivalent to $u(r)$ then either $s=r$ or $s=1-r$. It can be proved that the binomial relation is an equivalence relation. Some amount of calculation is needed to check that it is transitive. (For a proof, please refer to [5].)

DEFINITION. A number $r$ in the unit interval is said to be a binomial number provided that there exists $s$ in the unit interval such that $s$ is binomially related to $r$ and the number $s, r, 1-r$ are distinct. A binomial number is said to have of order $n$ if its equivalence class has exactly $n$ distinct elements.

A Selmer's number $r \in[-1,1]$ is an algebraic integer of degree $n>2$ so that $r^{n}-r-1=0$. In this article we show that if a Selmer's number $x$ has even degree, then $-x$ is a binomial number. Selmer proved, via continued fraction expressions, the irreducibility over $Q$ of the polynomials $(*) x^{n}-x-1$ in [7].

THEOREM 1. For $n>2$, there are algebraic integers of degree $n$ that are binomial numbers.

Proof. Case 1. $n$ is even, say $n=2 m, m>1$. Consider Selmer's algebraic integer $a$ of degree $n$ such that $a^{n}-a-1=0$ and $-1<a<0$. Let $r=-a$ and $s=r^{2}$. Then we have

$$
r=\sum_{1}^{m}\left(\begin{array}{l}
m \\
k
\end{array}\right) s^{m-k}(1-s)^{k} .
$$

Therefore $r$ is a binomial number of degree $n$.

Case 2. $n$ is odd, say $n=2 m+1, m>0$. According to the irreducible polynomial $x^{2 m+1}+x^{2 m}-1$, using $(*)$ and replacing $x$ by $1 / x$, there is an algebraic integer $r$ in the unit interval of degree $n$ so that if we set $s=r^{2}$, then

$$
r=\left(\sum_{\substack{k=0 \\
k \neq 1}}\left(\begin{array}{c}
m+1 \\
k
\end{array}\right) s^{m-k+1}(1-s)^{k}\right)+m s^{m}(1-s) .
$$

Furthermore $r, 1-r$, and $s$ are distinct. Therefore $r$ is a binomial number.

THEOREM 2. For each $n>2$, there are algebraic fractionals of degree $n$, that are binomial numbers.

PROOF. Case 1. $n=2 m$. The irreducibility of $2 x^{2 m}+2 x^{2 m-1}-x^{2}-x-1$ implies that there are algebraic fractionals of degree $n$ which are binomial numbers for each even integer $n>2$. 
I would like to thank Helge Tverberg in Bergen Norway for pointing out these irreducible polynomials.

Case 2. $n=2 m+1$. By using the irreducibility of

$$
2 x^{2 m+1}-2 x^{2 m}+2 x^{m}-1 \text {. }
$$

For details of the proof of Case 1 and Case 2, please refer to [5].

THEOREM 3. The equivalence class of $u(r)$ has two elements for each algebraic integer $r$ of degree 2 in the unit interval.

Proof. It suffices to show that $r$ is a non-binomial number. Let $[x]$ be the largest integer less than or equal to $x$ and $\langle x\rangle=x-[x]$. Then we have the following lemma.

LEMMA. Let $s$ be an irrational number and $m$ an integer with $|m|>1$. If $r=\langle m s\rangle$, then s can never be $\langle k r\rangle$ for any integer $k$.

Let $r$ be an algebraic integer of degree 2 and $s$ be binomially related to $r$. Then $r, s$ satisfy (1) and (2). Therefore $s$ is an algebraic integer of degree 2 and $s=\langle p r\rangle$ and $r=\langle q s\rangle$ for some integers $p, q$. Applying the preceding Lemma, we obtain $s=r$ or $s=1-r$.

There is another proof for Theorem 3 in [5].

REMARK. In a preliminary report of this abstract [6], it was asserted that there exist $r, s$ with $s \neq r, s \neq 1-r$ such that $u(s)$ and $u(r)$ are topologically equivalent. This abstract has subsequently been quoted by V. Prasad in [8]. The long and complicated proof of this assertion has been found to contain an error. I apologize here for this error. A generalization of this article had been studied in [5]. I wish to thank the referee for his helpful corrections of this article's earlier version. I am grateful to Dr. R. D. Mauldin for his suggestion of the style and the corrections of this revision.

\section{REFERENCES}

1. J. C. Oxtoby and S. M. Ulam, Measure preserving homeomorphisms and metrical transitivity, Ann. of Math. (2) 42 (1941), 874-920.

2. J. C. Oxtoby, Homeomorphic measures in metric space, Proc. Amer. Math. Soc. 24 (1970), 419-423.

3. J. C. Oxtoby and V. Prasad, Homeomorphic measures in the Hilbert cube, Pacific J. Math. 77 (1978), 483-497.

4. F. J. Navarro-Bermúdez, Topologically equivalent measures in the Cantor space, Proc. Amer. Math. Soc. 77 (1979), 229-236.

5. K. J. Huang, Algebraic numbers and topologically equivalent measures, Thesis, North Texas State University, 1983.

6. __ Topologically equivalent measures in the Cantor space, Abstracts Amer. Math. Soc. 2 (1981), 572.

7. Ernest S. Selmer, On the irreducibility of certain trinomials, Math. Scand. 4 (1956), 287302 .

8. D. Kölzow and D. Maharam-Stone (Eds.), Measure theory, Oberwolfach 1981, Proceedings; Lecture Notes in Math., vol. 945, Springer-Verlag, p. 153.

Department of Mathematics and Computer SCience, California State UniVERSITY, LOS ANGELES, CALIFORNIA 90032 